Mathematical Modelling and Analysis

Volume 13 Number 2, 2008, PAGes 183-194

(C) 2008 Technika ISSN 1392-6292 print, ISSN 1648-3510 online

\title{
DIFFRACTION BY A UNION OF STRIPS WITH IMPEDANCE CONDITIONS IN BESOV AND BESSEL POTENTIAL SPACES ${ }^{1}$
}

\author{
L. P. CASTRO and D. KAPANADZE \\ Research Unit "Matemática e Aplicações", Department of Mathematics, \\ University of Aveiro
}

3810-193 Aveiro, Portugal

E-mail: castro@ua.pt;david.kapanadze@gmail.com

Received August 6, 2007; revised December 17, 2007; published online May 1, 2008

\begin{abstract}
We consider an impedance boundary-value problem for the Helmholtz equation which models a wave diffraction problem with imperfect conductivity on a union of strips. Pseudo-differential operators acting between Bessel potential spaces and Besov spaces are used to deal with this wave diffraction problem. In particular, these operators allow a reformulation of the problem into a system of integral equations. The main result presents impedance parameters which ensure the wellposedness of the problem in scales of Bessel potential spaces and Besov spaces.
\end{abstract}

Key words: Wave diffraction, boundary-value problem, impedance conditions, pseudo-differential operators, Bessel potential spaces, Besov spaces.

\section{Introduction}

The diffraction of waves by regions in a plane is an important topic in scattering theory from both mathematical and engineering points of view, and has been subjected to numerous past investigations. We refer to the survey paper [6] and to the book [3] for a description on the background of these problems.

From the mathematical point of view (depending on the kind of boundary conditions in use and on the geometry of the problem), different studies have been made about the type of the spaces which are more appropriate to deal with such kind of problems (cf. $[1,2,6]$ and the references therein). In fact, a great part of the mathematical interest in this kind of problems is devoted to the question of finding out the largest set of possible spaces where the existence of a unique solution, and continuous dependence on the given data will be shown.

\footnotetext{
${ }^{1}$ This work was supported in part by Unidade de Investigação Matemática e Aplicações of Universidade de Aveiro through the Portuguese Science Foundation (FCT-Fundação para a Ciência e a Tecnologia).
} 
Within this goal, it is relevant to mention that for the real (non-complex) wave number case some of the known techniques fail (e.g., certain integral representations constructed by the Wiener-Hopf method for the complex wave number case have no sense in the real wave number situation).

The aim of this work is to analyse the problem of plane wave diffraction by a union of strips. The problem will be formulated in both cases of real and complex wave numbers, and worked out in a framework of Bessel potential and Besov spaces with general integrability and smoothness indices. In addition, impedance conditions will be assumed on both faces of the union of the strips. We will provide new results on the possible smoothness orders and integrability parameters of Bessel potential and Besov spaces for the well-posedness of the announced problem. Thus, the present work improve the known corresponding results in two directions: generalization of the geometrical situation (several strips instead of only one), and generalization of the spaces in consideration (having Bessel potential and Besov spaces with general smoothness and integrability indices). Therefore, we improve for instance the results of [2] where only the one strip geometry was considered and in Hilbert Bessel potential spaces.

\section{The Impedance Boundary Value Problem for the Union of Strips}

In this section we introduce the general notation, and present the mathematical formulation of the diffraction problem.

Let $m$ be a positive integer number. We will work within the framework of Bessel potential $H_{p}^{s}\left(\mathbb{R}^{m}\right)$ and Besov $B_{p, q}^{s}\left(\mathbb{R}^{m}\right)$ spaces (for $s \in \mathbb{R}, 1<p<\infty$, and $1 \leq q \leq \infty)$. We refer to [7] for the general properties of these spaces. For a given $\mathscr{D} \subset \mathbb{R}^{m}$, we will also work with $H_{p}^{s}(\mathscr{D})$, and denote by $\widetilde{H}_{p}^{s}(\mathscr{D})$ the closed subspace of $H_{p}^{s}\left(\mathbb{R}^{m}\right)$ whose elements have supports in $\overline{\mathscr{D}}$. The corresponding Besov spaces on $\mathscr{D}$ will be also used. In addition, we will consider the local Besov and Bessel potential spaces.

Let $n$ be a positive integer number. Considering the Cartesian axes Oxyz, we assume throughout this work that the material is invariant in the $z$-direction. Therefore, in effect, the geometry of the problem is two-dimensional, which leads us from strips to finite intervals. Thus, let us consider

$$
\Omega:=\mathbb{R}^{2} \backslash\left\{(x, 0) \in \mathbb{R}^{2} \mid x \in \bar{\Sigma}\right\},
$$

where $\Sigma:=] a_{0}, a_{1}[\cup \cdots \cup] a_{n-1}, a_{n}\left[\right.$ for $0=a_{0}<a_{1}<a_{2}<\cdots<a_{n}<\infty$, and study the problem of existence and uniqueness of an element $u \in L_{2}\left(\mathbb{R}^{2}\right)$, with $u_{\mid \Omega} \in H_{2, \text { loc }}^{1}(\Omega)$, such that

$$
\begin{aligned}
& \left(\frac{\partial^{2}}{\partial x^{2}}+\frac{\partial^{2}}{\partial y^{2}}+k^{2}\right) u=0 \quad \text { in } \quad \Omega, \\
& \left\{\begin{array}{l}
u_{1}^{+}-i \varrho^{+} u_{0}^{+}=h^{+} \\
u_{1}^{-}+i \varrho^{-} u_{0}^{-}=
\end{array} h^{-} \quad \text { on } \quad \Sigma,\right.
\end{aligned}
$$


where the wave number $k \neq 0$ is given, as well as $\varrho^{ \pm} \in \mathbb{C}$, and the Dirichlet and Neumann traces are denoted by $u_{0}^{ \pm}=u_{\mid y= \pm 0}$ and $u_{1}^{ \pm}=-(\partial u / \partial y)_{\mid y= \pm 0}$, respectively. The elements $h^{ \pm} \in H_{2}^{-1 / 2}(\Sigma)$ are arbitrarily given since the dependence on the data is to be studied for well-posedness. Note that the conditions in (2.2) are understood in the distributional sense.

Later on (as mentioned in the Introduction) we will also consider this problem in a larger framework where the solution is studied in both Bessel potential and Besov spaces within a larger range of integrability and smoothness space parameters.

We recall that we will deal with both situations of a dissipative and a non-dissipative medium reflected by the conditions $\Im \mathrm{m} k \neq 0$ and $\Im \mathrm{m} k=0$, respectively. Thus, for the real wave number case $(k \in \mathbb{R})$ it is natural to require that the eventual solution of (2.1)-(2.2) should also satisfy the Sommerfeld radiation condition at infinity

$$
u \in \operatorname{Som}(\Omega): \frac{\partial}{\partial|x|} u(x)-i|k| u(x)=\mathscr{O}\left(|x|^{-\frac{3}{2}}\right) \text { for }|x| \rightarrow \infty,
$$

see, e.g., [3]. Therefore, in what follows it is assumed that for a real $k$ we have a radiating $u$, while for a complex $k$ it follows that $u$ exponentially decays at infinity.

Let $r_{\Sigma}$ denote the restriction operator to $\Sigma$ (in corresponding spaces of Besov or Bessel potentials). Note that the conditions (2.1)-(2.2) lead to the following transmission conditions on $\mathbb{R} \backslash \bar{\Sigma}: u_{0}^{+}-u_{0}^{-}=0$ and $u_{1}^{+}-u_{1}^{-}=0$. Thus, when computing

$$
h^{+}-h^{-}=r_{\Sigma}\left(u_{1}^{+}-u_{1}^{-}\right)-r_{\Sigma}\left(i \varrho^{+} u_{0}^{+}+i \varrho^{-} u_{0}^{-}\right),
$$

we conclude that we necessarily need to have a compatibility condition between the data:

$$
h^{+}-h^{-} \in r_{\Sigma} \widetilde{H}_{2}^{-1 / 2}(\Sigma) .
$$

This occurs because from the transmission conditions on $\mathbb{R} \backslash \bar{\Sigma}$ and (2.2) it follows $u_{1}^{+}-u_{1}^{-} \in \widetilde{H}_{2}^{-1 / 2}(\Sigma)$, and also due to $i \varrho^{+} r_{\Sigma} u_{0}^{+}+i \varrho^{-} r_{\Sigma} u_{0}^{-} \in H_{2}^{1 / 2}(\Sigma)$ (which does not change the space in $(2.3)$ because $H_{2}^{1 / 2}(\Sigma) \hookrightarrow r_{\Sigma} \widetilde{H}_{2}^{-1 / 2}(\Sigma)$ is continuously embedded).

From now on we will refer to Problem $\mathscr{P}_{\text {Imp }}$ as the one characterized by (2.1), (2.2), (2.3) and the Sommerfeld radiation condition at infinity, in the case of $k \in \mathbb{R}$, and by (2.1), (2.2) and (2.3) in the case of $k \notin \mathbb{R}$.

\section{Uniqueness of Solution}

Let us assume that $\Sigma$ is a part of some smooth and simple curve $S$ which separates the space $\mathbb{R}^{2}$ into two disjoint domains $\Omega^{+}$and $\Omega^{-}=\mathbb{R}^{2} \backslash \overline{\Omega^{+}}$, such that $\Omega^{+}$is a bounded domain and $S=\partial \Omega^{ \pm}$(cf. Figure 1). In this case, we will denote by $n(z)=\left(n_{1}(z), n_{2}(z)\right)$ the outward unit normal vector at the point $z \in S=\partial \Omega^{+}$(see Figure 1$)$. 


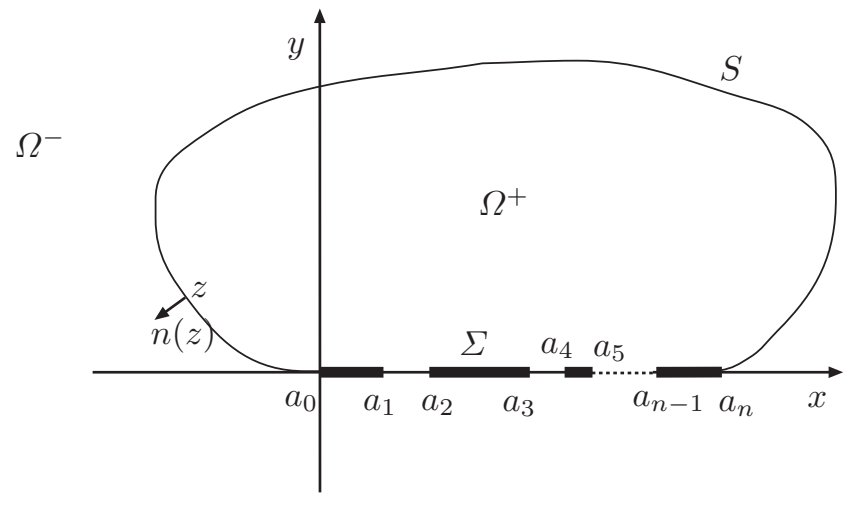

Figure 1. The geometry of the problem.

Theorem 1. (i) If $\Im \mathrm{m} k=0, \Re \mathrm{e} \varrho^{ \pm} \geq 0$, then the homogeneous Problem $\mathscr{P}_{\text {Imp }}$ (i.e., Problem $\mathscr{P}_{\text {Imp }}$ in the particular case of $h^{ \pm}=0$ ) has only the trivial solution $u=0$ in the space $H_{2, \operatorname{loc}}^{1}(\Omega) \cap \operatorname{Som}(\Omega)$.

(ii) If $\Im \mathrm{m} k \neq 0$, and one of the following situations holds:

(a) $\quad(\Re \mathrm{e} k)(\Im \mathrm{m} k)>0, \quad \Re \mathrm{e} \varrho^{ \pm} \geq 0$

(b) $\quad(\Re \mathrm{e} k)(\Im \mathrm{m} k)<0, \quad \Re \mathrm{e} \varrho^{ \pm} \leq 0$

(c) $\quad|\Im \mathrm{m} k| \geq|\Re \mathrm{e} k|, \quad \Im \mathrm{m} \varrho^{ \pm} \geq 0$

(d) $\quad \Re \mathrm{e} k=0, \quad \Re \mathrm{e} \varrho^{+} \neq 0, \quad \Im \mathrm{m} \varrho^{-} \geq\left(\Im \mathrm{m} \varrho^{+}\right)\left(\Re \mathrm{e} \varrho^{-}\right) /\left(\Re \mathrm{e} \varrho^{+}\right)$

(e) $\quad \Re \mathrm{e} k=0, \quad \Re \mathrm{e} \varrho^{-} \neq 0, \quad \Im \mathrm{m} \varrho^{+} \geq\left(\Im \mathrm{m} \varrho^{-}\right)\left(\Re \mathrm{e} \varrho^{+}\right) /\left(\Re \mathrm{e} \varrho^{-}\right)$

(f) $\quad \Re \mathrm{e} k \neq 0, \quad \varrho^{-}=0, \quad \Re \mathrm{e} \varrho^{+} \neq 0, \quad \Im \mathrm{m} \varrho^{+} \neq 0$,

$(\Im \mathrm{m} k)^{2}-(\Re \mathrm{e} k)^{2}-2(\Re \mathrm{e} k)(\Im \mathrm{m} k)\left(\Im \mathrm{m} \varrho^{+}\right) /\left(\Re \mathrm{e} \varrho^{+}\right) \geq 0$

(g) $\quad \Re \mathrm{e} k \neq 0, \quad \varrho^{+}=0, \quad \Re \mathrm{e} \varrho^{-} \neq 0, \quad \Im \mathrm{m} \varrho^{-} \neq 0$,

$(\Im \mathrm{m} k)^{2}-(\Re \mathrm{e} k)^{2}-2(\Re \mathrm{e} k)(\Im \mathrm{m} k)\left(\Im \mathrm{m} \varrho^{-}\right) /\left(\Re \mathrm{e} \varrho^{-}\right) \geq 0$,

then the homogeneous Problem $\mathscr{P}_{\text {Imp }}$ has only the trivial solution $u=0$ in the space $H_{2}^{1}(\Omega)$.

Proof. (i) Let us first consider the case of $\Im \mathrm{m} k=0, \Re \mathrm{e} \varrho^{ \pm} \geq 0$. Take $R$ to be a sufficiently large positive number and let $B(R)$ be the disk centered at the origin with radius $R$, such that $\overline{\Omega_{+}} \subset B(R)$. We define $\Omega_{R}^{-}:=\Omega^{-} \cap B(R)$, and consider $u$ to be a solution of the homogeneous problem $\mathscr{P}_{\text {Imp }}$. Then, Green's formula for $u$ and its complex conjugate $\bar{u}$ (in the domains $\Omega_{R}^{-}$and $\Omega^{+}$) yields

$$
\begin{aligned}
& \int_{\Omega_{R}^{-}}\left(|\nabla u|^{2}-k^{2}|u|^{2}\right) d x=-\left\langle\left[\partial_{n} u\right]_{S}^{-},[u]_{S}^{-}\right\rangle_{S}+\int_{\partial B(R)} \partial_{n} u \bar{u} d S \\
& \int_{\Omega^{+}}\left(|\nabla u|^{2}-k^{2}|u|^{2}\right) d x=\left\langle\left[\partial_{n} u\right]_{S}^{+},[u]_{S}^{+}\right\rangle_{S}
\end{aligned}
$$


where the symbols $[\cdot]^{ \pm}$denote the non-tangential limit values on $S$ from $\Omega^{ \pm}$ and $\langle\cdot, \cdot\rangle_{S},\langle\cdot, \cdot\rangle_{\Sigma}$ denote the duality brackets between the dual spaces $H_{2}^{-\frac{1}{2}}(S)$ and $H_{2}^{\frac{1}{2}}(S)$, or $\widetilde{H}_{2}^{-\frac{1}{2}}(\Sigma)$ and $H_{2}^{\frac{1}{2}}(\Sigma)$, or $H_{2}^{-\frac{1}{2}}(\Sigma)$ and $\widetilde{H}_{2}^{\frac{1}{2}}(\Sigma)$.

Note that the interior regularity in $\Omega$ of solutions of the Helmholtz equation (2.1) gives us $[u]_{S \backslash \bar{\Sigma}}^{+}=[u]_{S \backslash \bar{\Sigma}}^{-}$and $\left[\partial_{n} u\right]_{S \backslash \bar{\Sigma}}^{+}=\left[\partial_{n} u\right]_{S \backslash \bar{\Sigma}}^{-}$. Then, by summing up the last two integral equations given in $\Omega^{+}$and $\Omega_{R}^{-}$, we obtain

$$
\begin{gathered}
\int_{\Omega^{+} \cup \Omega_{R}^{-}}\left(|\nabla u|^{2}-k^{2}|u|^{2}\right) d x=\left\langle u_{1}^{+}, u_{0}^{+}\right\rangle_{\Sigma}-\left\langle u_{1}^{-}, u_{0}^{-}\right\rangle_{\Sigma}+\int_{\partial B(R)} \partial_{n} u \bar{u} d S \\
=i \varrho^{+}\left\langle u_{0}^{+}, u_{0}^{+}\right\rangle_{\Sigma}+i \varrho^{-}\left\langle u_{0}^{-}, u_{0}^{-}\right\rangle_{\Sigma}+\int_{\partial B(R)} \partial_{n} u \bar{u} d S .
\end{gathered}
$$

Now, since we are assuming the parameter $R$ to be sufficiently large, we can apply the Sommerfeld radiation condition on the circle $\partial B(R)$. Let us now separate the imaginary part of the equation (3.1), and use the fact that $u \in$ $\operatorname{Som}(\Omega)$ implies $u(x)=\mathscr{O}\left(|x|^{-\frac{1}{2}}\right)$ as $|x| \rightarrow \infty$. We therefore obtain

$$
\Re \mathrm{e} \varrho^{+} \int_{\Sigma}\left|u_{0}^{+}\right|^{2} d \Sigma+\Re \mathrm{e} \varrho^{-} \int_{\Sigma}\left|u_{0}^{-}\right|^{2} d \Sigma+|k| \int_{\partial B(R)}|u|^{2} d S=\mathscr{O}\left(R^{-1}\right),
$$

which yields $\lim _{R \rightarrow \infty} \int_{\partial B(R)}|u|^{2} d S=0$, due to the conditions $\Re$ e $\varrho^{ \pm} \geq 0$. Consequently, from the Rellich-Vekua Theorem [8], it follows that $u=0$ in $\Omega$.

(ii) For the second case, we can repeat the same reasoning as in the case (i) up to the step of formula (3.1). Additionally, since $\Im \mathrm{m} k \neq 0$, the solution $u \in H_{2}^{1}(\Omega)$ of the Helmholtz equation exponentially decays at infinity and so it follows

$$
\int_{\Omega}\left(|\nabla u|^{2}-k^{2}|u|^{2}\right) d x=i \varrho^{+}\left\langle u_{0}^{+}, u_{0}^{+}\right\rangle_{\Sigma}+i \varrho^{-}\left\langle u_{0}^{-}, u_{0}^{-}\right\rangle_{\Sigma},
$$

when passing (3.1) to the limit as $R \rightarrow \infty$. From the real and imaginary parts of the last identity, we obtain

$$
\begin{aligned}
& \int_{\Omega}\left(|\nabla u|^{2}+\left((\Im \mathrm{m} k)^{2}-(\Re \mathrm{e} k)^{2}\right)|u|^{2}\right) d x=-\Im \mathrm{m} \varrho^{+}\left\langle u_{0}^{+}, u_{0}^{+}\right\rangle_{\Sigma}-\Im \mathrm{m} \varrho^{-}\left\langle u_{0}^{-}, u_{0}^{-}\right\rangle_{\Sigma}, \\
& -2(\Re \mathrm{e} k)(\Im \mathrm{m} k) \int_{\Omega}|u|^{2} d x=\Re \mathrm{e} \varrho^{+}\left\langle u_{0}^{+}, u_{0}^{+}\right\rangle_{\Sigma}+\Re \mathrm{e} \varrho^{-}\left\langle u_{0}^{-}, u_{0}^{-}\right\rangle_{\Sigma} \cdot
\end{aligned}
$$

In conclusion, for each of the conditions (a)-(g), it follows from the last two identities that $u=0$ in $\Omega$.

\section{The Integral Form of the Solutions}

In the present section we will use pseudodifferential operators, acting between Bessel potential and Besov spaces, for studying the solution of the original problem. Without lost of generality, we will assume that $k>0$ when in the 
case of a real wave number (the complementary case of $k<0$ runs with obvious changes).

Let us denote the standard fundamental solution of the Helmholtz equation (in two dimensions) by $\Gamma(x, k):=-\frac{i}{4} \mathscr{H}_{0}^{(1)}(k|x|)$, where $\mathscr{H}_{0}^{(1)}(k|x|)$ is the Hankel function of the first kind of order zero. Recall that the fundamental function $\Gamma(x, k)$ satisfies the Sommerfeld radiation condition (for a real positive $k$ ), and it has the following logarithmic singularity in the neighbourhood of the origin $\Gamma(x, k)=-\frac{1}{2 \pi} \ln \frac{1}{|x|}+\mathscr{O}\left(|x|^{2} \ln |x|\right),|x|<\frac{1}{2}$ (cf. [3, §3.4]). Then the corresponding single and double layer potentials are of the form $V(\psi)(x)=$ $\int_{\Sigma} \Gamma(x-y, k) \psi(y) d S, x \notin \Sigma$, and $W(\varphi)(x)=\int_{\Sigma}\left[\partial_{n(y)} \Gamma(x-y, k)\right] \varphi(y) d S$, $x \notin \Sigma$, where $\psi$ and $\varphi$ are density functions.

Note that by the standard arguments of the Green identities we obtain the following integral representation of a radiating solution $u \in H_{2, \text { loc }}^{1}(\Omega) \cap \operatorname{Som}(\Omega)$ of the homogeneous Helmholtz equation (cf. [8])

$$
\begin{array}{r} 
\pm \int_{S}\left\{\left[\partial_{n(y)} \Gamma(x-y, k)\right][u(y)]^{ \pm}-\Gamma(x-y, k)\left[\partial_{n(y)} u(y)\right]^{ \pm}\right\} d S \\
=\left\{\begin{array}{lll}
u(x) & \text { for } & x \in \Omega^{ \pm} \\
0 & \text { for } & x \in \Omega^{\mp}
\end{array}\right.
\end{array}
$$

In addition, by summing up (and taking into account the continuity relations $[u]_{S \backslash \bar{\Sigma}}^{+}=[u]_{S \backslash \bar{\Sigma}}^{-}$and $\left.\left[\partial_{n} u\right]_{S \backslash \bar{\Sigma}}^{+}=\left[\partial_{n} u\right]_{S \backslash \bar{\Sigma}}^{-}\right)$, we derive that

$$
\begin{gathered}
u(x)=\int_{\Sigma}\left\{\left[\partial_{n(y)} \Gamma(x-y, k)\right]\left([u(y)]^{+}-[u(y)]^{-}\right)-\Gamma(x-y, k)\left(\left[\partial_{n(y)} u(y)\right]^{+}\right.\right. \\
\left.\left.-\left[\partial_{n(y)} u(y)\right]^{-}\right)\right\} d S=W\left(u_{0}^{+}-u_{0}^{-}\right)(x)-V\left(u_{1}^{+}-u_{1}^{-}\right)(x), \quad x \in \Omega, \quad(4.2
\end{gathered}
$$

which holds for a solution $u \in H_{2, \text { loc }}^{1}(\Omega) \cap \operatorname{Som}(\Omega)$ when $k$ is a positive real number, as well as for a solution $u \in H_{2}^{1}(\Omega)$ of the Helmholtz equation when $k$ is a complex (non-real) wave number.

Let us now recall some mapping properties of the above single and double layer potentials (cf., e.g., [4] and [5]), in Bessel potential and Besov spaces with indices $s \in \mathbb{R}, 1<p<\infty$, and $1 \leq q \leq \infty$ :

$$
\begin{array}{cl}
V: H_{2}^{s}(S) \rightarrow H_{2, \operatorname{loc}}^{s+\frac{3}{2}}\left(\Omega^{-}\right) \cap \operatorname{Som}\left(\Omega^{-}\right) & {\left[H_{2}^{s}(S) \rightarrow H_{2}^{s+\frac{3}{2}}\left(\Omega^{+}\right)\right],} \\
: B_{p, p}^{s}(S) \rightarrow H_{p, \operatorname{loc}}^{s+\frac{1}{p}}\left(\Omega^{-}\right) \cap \operatorname{Som}\left(\Omega^{-}\right) & {\left[B_{p, p}^{s}(S) \rightarrow H_{p}^{s+1+\frac{1}{p}}\left(\Omega^{+}\right)\right],} \\
: B_{p, q}^{s}(S) \rightarrow B_{p, q, \operatorname{loc}}^{s+1+\frac{1}{p}}\left(\Omega^{-}\right) \cap \operatorname{Som}\left(\Omega^{-}\right) & {\left[B_{p, q}^{s}(S) \rightarrow B_{p, q}^{s+1+\frac{1}{p}}\left(\Omega^{+}\right)\right],} \\
W: H_{2}^{s}(S) \rightarrow H_{2, \operatorname{loc}}^{s+\frac{1}{2}}\left(\Omega^{-}\right) \cap \operatorname{Som}\left(\Omega^{-}\right) & {\left[H_{2}^{s}(S) \rightarrow H_{2}^{s+\frac{1}{2}}\left(\Omega^{+}\right)\right]} \\
: B_{p, p}^{s}(S) \rightarrow H_{p, \operatorname{loc}}^{s+\frac{1}{p}}\left(\Omega^{-}\right) \cap \operatorname{Som}\left(\Omega^{-}\right) & {\left[B_{p, p}^{s}(S) \rightarrow H_{p}^{s+\frac{1}{p}}\left(\Omega^{+}\right)\right]} \\
: B_{p, q}^{s}(S) \rightarrow B_{p, q, \operatorname{loc}}^{s+\frac{1}{p}}\left(\Omega^{-}\right) \cap \operatorname{Som}\left(\Omega^{-}\right) & {\left[B_{p, q}^{s}(S) \rightarrow B_{p, q}^{s+\frac{1}{p}}\left(\Omega^{+}\right)\right]}
\end{array}
$$


for a real positive wave number $k$, and

$$
\begin{aligned}
V \quad: & H_{2}^{s}(S) \rightarrow H_{2}^{s+\frac{3}{2}}\left(\Omega^{ \pm}\right) & & : H_{2}^{s}(S) \rightarrow H_{2}^{s+\frac{1}{2}}\left(\Omega^{ \pm}\right) \\
: & B_{p, p}^{s}(S) \rightarrow H_{p}^{s+1+\frac{1}{p}}\left(\Omega^{ \pm}\right) & : & B_{p, p}^{s}(S) \rightarrow H_{p}^{s+\frac{1}{p}}\left(\Omega^{ \pm}\right) \\
: \quad & B_{p, q}^{s}(S) \rightarrow B_{p, q}^{s+1+\frac{1}{p}}\left(\Omega^{ \pm}\right) & : & B_{p, q}^{s}(S) \rightarrow B_{p, q}^{s+\frac{1}{p}}\left(\Omega^{ \pm}\right),
\end{aligned}
$$

for a complex (non-real) $k$. For $\psi \in \widetilde{H}_{2}^{-\frac{1}{2}}(S)$ and $\varphi \in \widetilde{H}_{2}^{\frac{1}{2}}(S)$, the following jump relations are also well-known

$$
\begin{array}{ll}
{[V(\psi)]_{S}^{+}=[V(\psi)]_{S}^{-}=: \mathscr{H}(\psi),} & {\left[\partial_{n} V(\psi)\right]_{S}^{ \pm}=:\left[\mp \frac{1}{2} I+\mathscr{K}\right](\psi),} \\
{[W(\varphi)]_{S}^{ \pm}=:\left[ \pm \frac{1}{2} I+\mathscr{K}^{*}\right](\varphi),} & {\left[\partial_{n} W(\varphi)\right]_{S}^{+}=\left[\partial_{n} W(\varphi)\right]_{S}^{-}=: \mathscr{L}(\varphi),}
\end{array}
$$

where $I$ denotes the identity operator, and

$$
\begin{aligned}
& \mathscr{H}(\psi)(z):=\int_{S} \Gamma(z-y, k) \psi(y) d S, \quad z \in S, \\
& \mathscr{K}(\psi)(z):=\int_{S}\left[\partial_{n(z)} \Gamma(z-y, k)\right] \psi(y) d S, \quad z \in S, \\
& \mathscr{K}^{*}(\varphi)(z):=\int_{S}\left[\partial_{n(y)} \Gamma(y-z, k)\right] \varphi(y) d S, \quad z \in S, \\
& \mathscr{L}(\varphi)(z):=\lim _{x \rightarrow z \in S} \partial_{n(x)} \int_{S}\left[\partial_{n(y)} \Gamma(y-x, k)\right] \varphi(y) d S, \quad z \in S .
\end{aligned}
$$

Note that in our geometry, after restricting the above $\mathscr{K}$ and $\mathscr{K}^{*}$ to $\Sigma$ they became to be zero operators and so they will not make significant influence in the invertibility study. Therefore, they are omitted in below considerations.

Theorem 2. Let $s \in \mathbb{R}, 1<p<\infty$, and $1 \leq q \leq \infty$. The operators (4.6) and (4.9) considered now on $\Sigma$ are pseudo-differential operators of order -1 and 1 (respectively) which can be restricted/extended to the following bounded mappings:

$$
\begin{array}{llll}
r_{\Sigma} \mathscr{H}: & \widetilde{H}_{p}^{s}(\Sigma) \rightarrow H_{p}^{s+1}(\Sigma) & {\left[\widetilde{B}_{p, q}^{s}(\Sigma) \rightarrow B_{p, q}^{s+1}(\Sigma)\right]} \\
r_{\Sigma} \mathscr{L}: & \widetilde{H}_{p}^{s+1}(\Sigma) \rightarrow H_{p}^{s}(\Sigma) & {\left[\widetilde{B}_{p, q}^{s+1}(\Sigma) \rightarrow B_{p, q}^{s}(\Sigma)\right] .}
\end{array}
$$

In addition:

(i) The operator $r_{\Sigma} \mathscr{H}: \widetilde{H}_{p}^{s}(\Sigma) \rightarrow H_{p}^{s+1}(\Sigma)$ is Fredholm if and only if

$$
\frac{1}{p}-\frac{3}{2}<s<\frac{1}{p}-\frac{1}{2} \quad(\text { and } \quad 1 \leq q \leq \infty) .
$$

(ii) The operator $r_{\Sigma} \mathscr{L}: \widetilde{H}_{p}^{s+1}(\Sigma) \rightarrow H_{p}^{s}(\Sigma)$ is Fredholm if and only if (4.12) holds true. 
(iii) The operator $r_{\Sigma} \mathscr{H}: \widetilde{B}_{p, q}^{s}(\Sigma) \rightarrow B_{p, q}^{s+1}(\Sigma)$ is Fredholm if (4.12) holds true.

(iv) The operator $r_{\Sigma} \mathscr{L}: \widetilde{B}_{p, q}^{s+1}(\Sigma) \rightarrow B_{p, q}^{s}(\Sigma)$ is Fredholm if (4.12) holds true.

(v) All the operators in (4.10) and (4.11) are invertible provided that (4.12) holds true.

The last result was derived using the methods detailed presented in $[5, \S 5]$ (the proof is omitted for a matter of brevity).

\section{Existence and Regularity of Solutions on Bessel Poten- tial and Besov Spaces}

In the present section, for the spaces $H_{p}^{s}$ or $B_{p, q}^{s}$, with $1 \leq s=1+\epsilon<2$, $1<p<\infty$, and $1 \leq q \leq \infty$, we will analyze the existence of a solution of the corresponding Problem $\mathscr{P}_{\text {Imp }}$, in the above indicated form

$$
u(x)=W(\varphi)(x)-V(\psi)(x), \quad x \in \Omega,
$$

where the unknown densities $\varphi$ and $\psi$ are related to the source $u$ and its normal derivative by the following equations (cf. (4.2)):

$$
\varphi=u_{0}^{+}-u_{0}^{-}, \quad \psi=u_{1}^{+}-u_{1}^{-} .
$$

The boundary conditions (2.2) (together with (2.3)) can be equivalently rewritten in the form

$$
\left\{\begin{array}{lll}
u_{1}^{+}-u_{1}^{-}-i \varrho^{+} u_{0}^{+}-i \varrho^{-} u_{0}^{-} & =f_{0} \\
u_{1}^{+}-i \varrho^{+} u_{0}^{+} & =f_{1}
\end{array} \quad \text { on } \Sigma,\right.
$$

where $f_{0}:=h^{+}-h^{-} \in r_{\Sigma} \widetilde{H}_{p}^{\epsilon-\frac{1}{p}}(\Sigma)\left[r_{\Sigma} \widetilde{B}_{p, q}^{\epsilon-\frac{1}{p}}(\Sigma)\right]$ and $f_{1}:=h^{+} \in H_{p}^{\epsilon-\frac{1}{p}}(\Sigma)$ $\left[B_{p, q}^{\epsilon-\frac{1}{p}}(\Sigma)\right]$, with $0 \leq \epsilon<1$. Then, the representation formula (5.1) together with the jump relations (4.5) and the boundary conditions (5.3) lead to the following system of equations on $\Sigma$ with unknown $\varphi$ and $\psi$ :

$$
\begin{cases}r_{\Sigma}\left\{\psi I-i \varrho^{+}\left[\frac{1}{2} I \varphi-\mathscr{H} \psi\right]-i \varrho^{-}\left[-\frac{1}{2} I \varphi-\mathscr{H} \psi\right]\right\} & =f_{0} \\ r_{\Sigma}\left\{\mathscr{L} \varphi+\frac{1}{2} I \psi-i \varrho^{+}\left[\frac{1}{2} I \varphi-\mathscr{H} \psi\right]\right\} & =f_{1} .\end{cases}
$$

For the matter of notation abbreviation, let us introduce the operator

$$
\mathscr{A}:=\left(\begin{array}{cc}
I+i \varrho^{+} \mathscr{H}+i \varrho^{-} \mathscr{H} & -i \varrho^{+}\left(\frac{1}{2} I\right)+i \varrho^{-}\left(\frac{1}{2} I\right) \\
\frac{1}{2} I+i \varrho^{+} \mathscr{H} & \mathscr{L}-i \varrho^{+}\left(\frac{1}{2} I\right)
\end{array}\right) \text {, }
$$

and $\Phi:=(\psi, \varphi)^{\top}, F:=\left(f_{0}, f_{1}\right)^{\top}$. Then, from (5.4), we have

$$
r_{\Sigma} \mathscr{A} \Phi=F \quad \text { on } \quad \Sigma
$$


where

$$
\Phi \in \widetilde{H}_{p}^{\epsilon-\frac{1}{p}}(\Sigma) \times \widetilde{H}_{p}^{\epsilon+1-\frac{1}{p}}(\Sigma)\left[\widetilde{B}_{p, q}^{\epsilon-\frac{1}{p}}(\Sigma) \times \widetilde{B}_{p, q}^{\epsilon+1-\frac{1}{p}}(\Sigma)\right]
$$

and

$$
F \in\left(r_{\Sigma} \widetilde{H}_{p}^{\epsilon-\frac{1}{p}}(\Sigma)\right) \times H_{p}^{\epsilon-\frac{1}{p}}(\Sigma)\left[\left(r_{\Sigma} \widetilde{B}_{p, q}^{\epsilon-\frac{1}{p}}(\Sigma)\right) \times B_{p, q}^{\epsilon-\frac{1}{p}}(\Sigma)\right] .
$$

Theorem 3. Let $\epsilon=0,1<p<\infty$, and $1 \leq q \leq \infty$.

(i) If $\Im \mathrm{m} k=0$ and $\Re \mathrm{e} \varrho^{ \pm} \geq 0$, then Problem $\mathscr{P}_{\text {Imp }}$ has a unique solution in the space $H_{p, \text { loc }}^{1}(\Omega) \cap \operatorname{Som}(\Omega)\left[B_{p, q, \text { loc }}^{1}(\Omega) \cap \operatorname{Som}(\Omega)\right]$, which is representable in the form (5.1) with the densities $\varphi$ and $\psi$ defined by the uniquely solvable pseudo-differential equation (5.5).

(ii) If $\Im \mathrm{m} k \neq 0$ and one of the conditions (a)-(g) of Theorem 1 is satisfied, then Problem $\mathscr{P}_{\text {Imp }}$ has a unique solution in the space $H_{p}^{1}(\Omega)\left[B_{p, q}^{1}(\Omega)\right]$, which is representable in the form (5.1) with the densities $\varphi$ and $\psi$ defined by the uniquely solvable pseudo-differential equation (5.5).

Proof. We will analyse the invertibility of the matrix operator

$$
\begin{aligned}
r_{\Sigma} \mathscr{A}: & \widetilde{H}_{p}^{-\frac{1}{p}}(\Sigma) \times \widetilde{H}_{p}^{1-\frac{1}{p}}(\Sigma) \rightarrow\left(r_{\Sigma} \widetilde{H}_{p}^{-\frac{1}{p}}(\Sigma)\right) \times H_{p}^{-\frac{1}{p}}(\Sigma) \\
: & \widetilde{B}_{p, q}^{-\frac{1}{p}}(\Sigma) \times \widetilde{B}_{p, q}^{1-\frac{1}{p}}(\Sigma) \rightarrow\left(r_{\Sigma} \widetilde{B}_{p, q}^{-\frac{1}{p}}(\Sigma)\right) \times B_{p, q}^{-\frac{1}{p}}(\Sigma) .
\end{aligned}
$$

Due the compact embeddings $\widetilde{H}_{p}^{1-1 / p}(\Sigma) \hookrightarrow \widetilde{H}_{p}^{-1 / p}(\Sigma), \widetilde{B}_{p, q}^{1-1 / p}(\Sigma) \hookrightarrow \widetilde{B}_{p, q}^{-1 / p}(\Sigma)$, $H_{p}^{1-1 / p}(\Sigma) \hookrightarrow r_{\Sigma} \widetilde{H}_{p}^{-1 / p}(\Sigma), B_{p, q}^{1-1 / p}(\Sigma) \hookrightarrow r_{\Sigma} \widetilde{B}_{p, q}^{-1 / p}(\Sigma)$, and to the mapping properties (4.10), the operators in use

$$
\begin{aligned}
& r_{\Sigma} I: \widetilde{H}_{p}^{1-\frac{1}{p}}(\Sigma) \rightarrow r_{\Sigma} \widetilde{H}_{p}^{-\frac{1}{p}}(\Sigma), \quad r_{\Sigma} I: \widetilde{H}_{p}^{1-\frac{1}{p}}(\Sigma) \rightarrow H_{p}^{-\frac{1}{p}}(\Sigma), \\
& r_{\Sigma} I: \widetilde{B}_{p, q}^{1-\frac{1}{p}}(\Sigma) \rightarrow r_{\Sigma} \widetilde{B}_{p, q}^{-\frac{1}{p}}(\Sigma), \quad r_{\Sigma} I: \widetilde{B}_{p, q}^{1-\frac{1}{p}}(\Sigma) \rightarrow B_{p, q}^{-\frac{1}{p}}(\Sigma), \\
& r_{\Sigma} \mathscr{H}: \widetilde{H}_{p}^{-\frac{1}{p}}(\Sigma) \rightarrow r_{\Sigma} \widetilde{H}_{p}^{-\frac{1}{p}}(\Sigma), \quad r_{\Sigma} \mathscr{H}: \widetilde{B}_{p, q}^{-\frac{1}{p}}(\Sigma) \rightarrow r_{\Sigma} \widetilde{B}_{p, q}^{-\frac{1}{p}}(\Sigma)
\end{aligned}
$$

are compact. Therefore, (5.6) is a compact perturbation of a triangular matrix operator with the invertible operators $r_{\Sigma} I$ and $r_{\Sigma} \mathscr{L}$ in the main diagonal (cf. (5.7) below). Consequently, (5.6) is a Fredholm operator with zero index. Then Theorem 1 implies that $\operatorname{Ker} r_{\Sigma \mathscr{A}}=\{0\}$, and therefore the equation (5.5) is uniquely solvable for arbitrary $F \in\left(r_{\Sigma} \widetilde{H}_{p}^{-\frac{1}{p}}(\Sigma)\right) \times H_{p}^{-\frac{1}{p}}(\Sigma)\left[\left(r_{\Sigma} \widetilde{B}_{p, q}^{-\frac{1}{p}}(\Sigma)\right) \times\right.$ $\left.B_{p, q}^{-\frac{1}{p}}(\Sigma)\right]$

Theorem 4. Let (i) $\Im \mathrm{m} k=0$ and $\Re \mathrm{e} \varrho^{ \pm} \geq 0$, or (ii) $\Im \mathrm{m} k \neq 0$ and one of the conditions (a)-(g) of Theorem 1 be satisfied. If the boundary data satisfy the conditions $\left(h^{+}, h^{-}\right) \in H_{p}^{-\frac{1}{p}+\epsilon}(\Sigma) \times H_{p}^{-\frac{1}{p}+\epsilon}(\Sigma)\left[B_{p, q}^{-\frac{1}{p}+\epsilon}(\Sigma) \times B_{p, q}^{-\frac{1}{p}+\epsilon}(\Sigma)\right]$, $h^{+}-h^{-} \in r_{\Sigma} \widetilde{H}_{p}^{-\frac{1}{p}+\epsilon}(\Sigma)\left[r_{\Sigma} \widetilde{B}_{p, q}^{-\frac{1}{p}+\epsilon}(\Sigma)\right]$, for $0 \leq \epsilon<\frac{2}{p}-\frac{1}{2}$ and $2 \leq p<4$ (see Figure 2), then the solution $u$ of the corresponding Problem $\mathscr{P}_{\text {Imp }}$ possesses the following regularity

$$
\begin{aligned}
& u \in H_{p, \operatorname{loc}}^{1+\epsilon}(\Omega) \cap \operatorname{Som}(\Omega) \quad\left[B_{p, q, \text { loc }}^{1+\epsilon}(\Omega) \cap \operatorname{Som}(\Omega)\right], \quad \text { in the case }(\mathrm{i}), \\
& u \in H_{p}^{1+\epsilon}(\Omega)\left[B_{p, q}^{1+\epsilon}(\Omega)\right], \quad \text { in the case }(\mathrm{ii}) .
\end{aligned}
$$




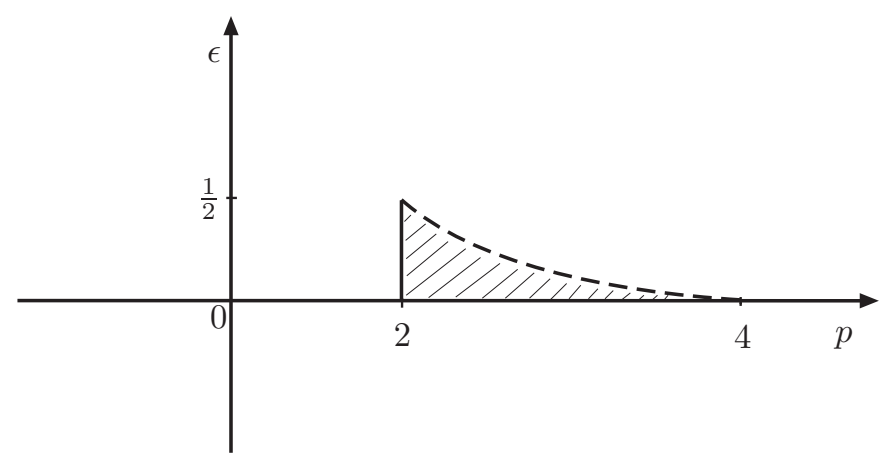

Figure 2. The regularity and integrability index region.

Proof. The solvability result follows from Theorem 3 . As for the regularity result, due to the continuity results in $(4.3)-(4.4)$, and the representation formula, it is sufficient to show that

$$
(\psi, \varphi) \in \widetilde{H}_{p}^{-\frac{1}{p}+\epsilon}(\Sigma) \times \widetilde{H}_{p}^{1-\frac{1}{p}+\epsilon}(\Sigma)\left[\widetilde{B}_{p, q}^{-\frac{1}{p}+\epsilon}(\Sigma) \times \widetilde{B}_{p, q}^{1-\frac{1}{p}+\epsilon}(\Sigma)\right]
$$

The operator $\mathscr{A}$ can be written as $\mathscr{A}=\mathscr{B}+T$, where

$$
\begin{aligned}
& \mathscr{B}:=\left(\begin{array}{cc}
I & 0 \\
\frac{1}{2} I & \mathscr{L}
\end{array}\right), \\
& T=\left(T_{j l}\right)_{j, l=1,2}:=\left(\begin{array}{cc}
i\left(\varrho^{+}+\varrho^{-}\right) \mathscr{H} & \frac{i}{2}\left(\varrho^{-}-\varrho^{+}\right) I \\
i \varrho^{+} \mathscr{H} & -\frac{i}{2} \varrho^{+} I
\end{array}\right) .
\end{aligned}
$$

Due to Theorem 2, we have that

$$
\begin{aligned}
r_{\Sigma} \mathscr{B} & : \quad \widetilde{H}_{p}^{-\frac{1}{p}+\epsilon}(\Sigma) \times \widetilde{H}_{p}^{1-\frac{1}{p}+\epsilon}(\Sigma) \rightarrow\left(r_{\Sigma} \widetilde{H}_{p}^{-\frac{1}{p}+\epsilon}(\Sigma)\right) \times H_{p}^{-\frac{1}{p}+\epsilon}(\Sigma) \\
: & \widetilde{B}_{p, q}^{-\frac{1}{p}+\epsilon}(\Sigma) \times \widetilde{B}_{p, q}^{1-\frac{1}{p}+\epsilon}(\Sigma) \rightarrow\left(r_{\Sigma} \widetilde{B}_{p, q}^{-\frac{1}{p}+\epsilon}(\Sigma)\right) \times B_{p, q}^{-\frac{1}{p}+\epsilon}(\Sigma)
\end{aligned}
$$

is an invertible operator for every $\epsilon \in\left(\frac{2}{p}-\frac{3}{2}, \frac{2}{p}-\frac{1}{2}\right), 1<p<\infty$ and $1 \leq q \leq \infty$. Indeed, the inverse is provided by the formula

$$
\left(r_{\Sigma} \mathscr{B}\right)^{-1}=\left(\begin{array}{cc}
\ell_{0} & 0 \\
-\frac{1}{2}\left(r_{\Sigma} \mathscr{L}\right)^{-1} & \left(r_{\Sigma} \mathscr{L}\right)^{-1}
\end{array}\right) .
$$

We will now study the operator $T$. When $\varrho^{-}+\varrho^{+}=0$ and $/$ or $\varrho^{+}=0$, some of the entries of the matrix operator $T$ are zero, and the below considerations are even simpler. In here, we will therefore consider in detail the most usual case when all entries are different from zero. 
Due to the mapping properties of Theorem 2, and to the compact embeddings

$$
\begin{aligned}
& H_{p}^{1-1 / p+\epsilon}(\Sigma) \hookrightarrow r_{\Sigma} \widetilde{H}_{p}^{\epsilon-1 / p}(\Sigma), \quad B_{p, q}^{1-1 / p+\epsilon}(\Sigma) \hookrightarrow r_{\Sigma} \widetilde{B}_{p, q}^{\epsilon-1 / p}(\Sigma), \\
& r_{\Sigma} \widetilde{H}_{p}^{1-1 / p+\epsilon}(\Sigma) \hookrightarrow H_{p}^{\epsilon-1 / p}(\Sigma), \quad r_{\Sigma} \widetilde{B}_{p, q}^{1-1 / p+\epsilon}(\Sigma) \hookrightarrow B_{p, q}^{\epsilon-1 / p}(\Sigma),
\end{aligned}
$$

it turns out that the operator $r_{\Sigma} T$ is compact. Moreover, the entries $T_{11}$, $T_{12}, T_{21}$, and $T_{22}$ are pseudo-differential operators of orders $-1,0,-1$ and 0 , respectively (see, Theorem 2 ).

Therefore, from $r_{\Sigma} \mathscr{A} \Phi=F$, we obtain $\Phi=\left(r_{\Sigma} \mathscr{B}\right)^{-1} F-\left(r_{\Sigma} \mathscr{B}\right)^{-1} r_{\Sigma} T \Phi$, i.e.,

$$
\begin{gathered}
\psi=\ell_{0} f_{0}-\ell_{0}\left(r_{\Sigma} T_{11} \psi-r_{\Sigma} T_{12} \varphi\right) \\
\varphi=\left(r_{\Sigma} \mathscr{L}\right)^{-1}\left(-\frac{1}{2} f_{0}+f_{1}\right)-\left(r_{\Sigma} \mathscr{L}\right)^{-1}\left(r_{\Sigma} T_{21}-\frac{1}{2} r_{\Sigma} T_{11}\right) \psi \\
\quad-\left(r_{\Sigma} \mathscr{L}\right)^{-1}\left(r_{\Sigma} T_{22}-\frac{1}{2} r_{\Sigma} T_{12}\right) \varphi .
\end{gathered}
$$

The last equality for $\psi$, together with

$$
(\psi, \varphi) \in \widetilde{H}_{p}^{-\frac{1}{p}}(\Sigma) \times \widetilde{H}_{p}^{1-\frac{1}{p}}(\Sigma)\left[\widetilde{B}_{p, q}^{-\frac{1}{p}}(\Sigma) \times \widetilde{B}_{p, q}^{1-\frac{1}{p}}(\Sigma)\right]
$$

and the continuity properties of $r_{\Sigma} T_{11}$ and $r_{\Sigma} T_{12}$, imply $\psi \in \widetilde{H}_{p}^{-\frac{1}{p}+\epsilon}(\Sigma)$ $\left[\widetilde{B}_{p, q}^{-\frac{1}{p}+\epsilon}(\Sigma)\right]$. Taking into account this result for the last equality of $\varphi$, and noting that $\mathscr{L}^{-1}$ is a pseudo-differential operator of order -1 , we obtain $\varphi \in \widetilde{H}_{p}^{1-\frac{1}{p}+\epsilon}(\Sigma)\left[\widetilde{B}_{p, q}^{1-\frac{1}{p}+\epsilon}(\Sigma)\right]$ and this concludes the proof.

\section{Acknowledgement}

D. Kapanadze acknowledge the support from the Portuguese Science Foundation (FCT-Fundação para a Ciência e a Tecnologia) through the grant number $\mathrm{SFRH} / \mathrm{BPD} / 20524 / 2004$.

\section{References}

[1] L.P. Castro and D. Kapanadze. Dirichlet-Neumann-impedance boundary-value problems arising in rectangular wedge diffraction problems. Proc. Am. Math. Soc., 136:2113-2123, 2008.

[2] L.P. Castro and D. Kapanadze. The impedance boundary-value problem of diffraction by a strip. J. Math. Anal. Appl., 337:1031-1040, 2008.

[3] D. Colton and R. Kress. Inverse Acoustic and Electromagnetic Scattering Theory. Springer-Verlag, Berlin, 1998.

Math. Model. Anal., 13(2):183-194, 2008. 
[4] R. Duduchava, D. Natroshvili and E. Shargorodsky. Boundary value problems of the mathematical theory of cracks. Proc. I. Vekua Inst. Appl. Math. (Tbilisi State University), 39:68-84, 1990.

[5] R. Duduchava, D. Natroshvili and E. Shargorodsky. Basic boundary value problems of thermoelasticity for anisotropic bodies with cuts, I and II. Georgian Math. J., 2:123-140 and 259-276, 1995.

[6] E. Meister and F.-O. Speck. Modern Wiener-Hopf methods in diffraction theory. Pitman Res. Notes Math. Ser., 216:130-171, 1989.

[7] H. Triebel. Interpolation Theory, Function Spaces, Differential Operators (2nd ed.). Barth, Leipzig, 1995.

[8] I.N. Vekua. On metaharmonic functions. Trudy Tbilisskogo Matematicheskogo Instituta, 12:105-174, 1943. 